\title{
Systemic Lupus Erythematosus Presenting with Thrombotic- Thrombocytopenic Purpura-like Syndrome and Catatonia
}

\author{
Manish Rathi ${ }^{1}$, Gaurav Vohra ${ }^{2}$, Jasmine Sethi ${ }^{3}$, Sandeep Grover ${ }^{4}$, Krishan L Gupta ${ }^{5}$
}

\begin{abstract}
Systemic lupus erythematosus (SLE) can present with thrombotic microangiopathy (TMA), and differentiating this from thrombotic thrombocytopenic purpura can be a challenging task. Though thrombotic-thrombocytopenic purpura (TTP) is a type of TMA demonstrating a severely reduced activity of VWF, cleaving protease ADAMTS 13 can differentiate it from other causes of TMA. Association of TTP-like TMA and catatonia in lupus is not described in literature. Neuropsychiatric lupus presenting as catatonia is uncommon. Here we describe a 41 -year-old female who was presented with TTP-like TMA and catatonic symptoms, which improved with plasma-exchange, benzodiazepines, and immunosuppression. Keywords: Catatonia, Systemic lupus erythematosus, Thrombotic microangiopathy, Thrombotic-Thrombocytopenic purpura.

Journal of Postgraduate Medicine Education and Research (2019): 10.5005/jp-journals-10028-1332
\end{abstract}

\section{BACKGROUND}

Thrombotic-thrombocytopenic purpura (TTP)-like thrombotic microangiopathy (TMA) syndrome can complicate a case of active lupus and responds favorably to plasma exchange and hiking of immunosuppression. TTP-like syndrome in lupus is recently identified as a new entity distinct from TTP. ${ }^{1}$ However, a clinician should have a high index of suspicion to suspect this rare entity early in the course to have a survival benefit. Catatonia can rarely occur in SLE and it is important to exclude central nervous system (CNS) infections. ${ }^{2}$ We report a case of TTP-like syndrome along with catatonia who survived with plasma-exchange and immunosuppressive therapy. In this study, we discuss the approach, differential diagnosis, and difficulties faced in managing such a case.

\section{Case Description}

A 41-year-old married female was presented to our outpatient clinic, with the complaints of anasarca, joint pains (small and large), and hair loss. On evaluation, we noted serum creatinine of $1.8 \mathrm{mg} / \mathrm{dL}$ and serum albumin of $1.8 \mathrm{~g} / \mathrm{dL}$; urine microscopy revealed albumin $2+$ and 2-3 RBC/Hpf, 24 hours urine protein of $3.2 \mathrm{~g} /$ day, low serum complements, positive antinuclear (ANA), and anti DsDNA antibody. Renal biopsy was consistent with lupus nephritis class 4 (G) with predominant active lesions and no evidence of thrombotic microangiopathy. She did not had any extrarenal manifestations at this time and was managed with three-day course of pulse methylprednisolone $(750 \mathrm{mg}$ ) followed by oral prednisolone at $1 \mathrm{mg} / \mathrm{kg}$ body weight. As a part of $\mathrm{NIH}$ protocol, first pulse of cyclophosphamide (500 mg) was administered.

The patient was admitted again a month later with worsening anasarca. Lab evaluation showed hemoglobin fall to a nadir of 5.7 $\mathrm{g} \%$ (from $9 \mathrm{~g} / \mathrm{dL}$ on presentation) and platelet count fell to nadir of 20,000/cu mm (from 1.5 lakh/cu $\mathrm{mm}$ ) requiring five units of blood and multiple random donor platelet transfusion. She had no history of oliguria, fever, joint pains, bleeding manifestations, shortness of breath, or hemoptysis. Her serum creatinine also increased to 4.8 $\mathrm{mg} / \mathrm{dL}$ (1.8 $\mathrm{mg} / \mathrm{dL}$ at baseline). Clinical suspicion of lupus activity in view of cytopenias, worsening renal function, and low complement levels was considered. Further evaluation revealed elevated lactate
1-3,5 Department of Nephrology, Postgraduate Institute of Medical Education and Research, Chandigarh, India

${ }^{4}$ Department of Psychiatry, Postgraduate Institute of Medical Education and Research, Chandigarh, India

Corresponding Author: Manish Rathi, Department of Nephrology, Postgraduate Institute of Medical Education and Research, Chandigarh, India, Phone: 0172-2756734, e-mail: drmanishrathi2000@yahoo.co.in

How to cite this article: Rathi M, Vohra G, Sethi J, et al. Systemic Lupus Erythematosus Presenting with Thrombotic-Thrombocytopenic Purpura-like Syndrome and Catatonia. J Postgrad Med Edu Res 2019;53(4):158-159.

Source of support: Nil

Conflict of interest: None

dehydrogenase of $1000 \mathrm{lU} / \mathrm{mL}$, repeated peripheral blood smear examinations showed $1-2 \%$ fragmentation index (normal $<0.5 \%$ ) with mild indirect hyperbilirubinimea (total bilirubin $2 \mathrm{mg} / \mathrm{dL}$, indirect bilirubin $1.3 \mathrm{mg} / \mathrm{dL}$ ) with positive direct Coombs test. White cell count, coagulogram, anticardiolipin antibody, and Lupus anticoagulant were in the normal range. Bone marrow examination revealed hypercellular marrow elements. After admission, she developed abnormal behavior in the form of self muttering, not responding, staring, mask-like facies, and running away from ward. In view of pentad of renal dysfunction, neurological involvement, features of hemolysis, thrombocytopenia, and fever, a clinical suspicion of TTP was considered and plasma-exchange was initiated along with a second pulse of cyclophosphamide (750 mg). CSF analysis and noncontrast MRI brain were done, which were normal. ADAMTS 13 activity levels could not be done owing to logistic issues. Possibilities considered were steroid-induced psychosis and neuropsychiatric lupus. Psychiatric consultation was taken and lorazepam challenge test was performed, which was positive, suggesting catatonia. For her catatonic symptoms, lorazepam was started orally at a dose of $2 \mathrm{mg} /$ day in three divided doses (total 6 $\mathrm{mg} /$ day). Following a course of fourteen plasmapheresis, her platelet count recovered to more than $100 \times 10^{9} / \mathrm{L}$, her fragmentation index decreased to below $1 \%$, and her hemoglobin stabilized to around $9 \mathrm{~g} / \mathrm{L}$. Serum creatinine decreased to $1.1 \mathrm{mg} / \mathrm{dL}$ and cytopenias

() The Author(s). 20190pen Access This article is distributed under the terms of the Creative Commons Attribution 4.0 International License (https://creativecommons. org/licenses/by-nc/4.0/), which permits unrestricted use, distribution, and non-commercial reproduction in any medium, provided you give appropriate credit to the original author(s) and the source, provide a link to the Creative Commons license, and indicate if changes were made. The Creative Commons Public Domain Dedication waiver (http://creativecommons.org/publicdomain/zero/1.0/) applies to the data made available in this article, unless otherwise stated. 
improved. The patient showed significant improvement with the above treatment and was discharged. She presently has no neuropsychiatric manifestations and maintains stable platelet count $\left(1.4 \mathrm{lakh} / \mathrm{mm}^{3}\right)$ and hemoglobin $(8.9 \mathrm{~g} / \mathrm{dL})$ at 4 weeks of follow-up.

\section{Discussion}

Neuropsychiatric manifestations are common in SLE (NPSLE) with a prevalence of $80-90 \%$ with headache as the most common feature. ${ }^{3,4}$ However, catatonia is rarely described and very few cases have been reported in literature. ${ }^{5-7}$ Catatonia can be seen at the disease onset or at relapse. ${ }^{7}$ A positive lorazepam challenge test establishes the diagnosis of catatonia as done in index patients. In this test, intravenous lorazepam is administered at a dose of $1-2 \mathrm{mg}$ and the patient is examined. Positive response is a marked reduction (50\%) of catatonic signs and symptoms by a standard rating scale. ${ }^{8}$ Most of the cases respond to lorazepam as seen in index case and by increasing dose of cytotoxic agent. ${ }^{9}$ Differentials for NPSLE include functional and steroid-induced psychosis. Our patient had no significant stressor apart from exacerbation of SLE; hence, a functional cause was unlikely. Steroid-induced psychiatric disturbance usually consists of mania, depression, and is typically dose-dependent, occurring in the first 1-2 weeks of starting steroids. ${ }^{10}$ In the index case, the patient was on steroid therapy for more than one month with nonaffective symptoms, hence unlikely to be steroid induced. Our case had TTP-like presentation in addition to catatonia, which is not described in literature till now. Whether catatonia was a part of TTP or separate neuropsychiatric involvement in this patient is difficult to comment.

Microangiopathic hemolytic anemia (MAHA) is a term used for nonimmune hemolysis resulting from intravascular red blood cell fragmentation, producing schistocytes on peripheral blood. Thrombotic microangiopathy is a pathological diagnosis based on the finding of microvascular thrombosis. However, a clinical combination of MAHA and thrombocytopenia suggests TMA." Differentials of thrombotic microangiopathy in a patient of SLE are described in Table 1. The two differentials considered upfront in the index case were sepsis with disseminated intravascular coagulation and cyclophosphamide-induced cytopenias. However, normal coagulation parameters, normal procalcitonin, and negative cultures ruled out infection. Bone marrow suggestive of hypercellular marrow with the evidence of microangiopathy pointed more in favor of TMA than drug-induced cytopenias.

Patients with lupus are at a particular risk for acquiring the anti-phospholipid syndrome, malignant hypertension, and

Table 1: Differentials of a patient of lupus with TMA

\begin{tabular}{ll}
\hline - & TTP \\
- & Dissug-induced TMA \\
- & Catastrophic antiphopholipid syndrome (CAPS) \\
- & Malignant hypertension \\
- & Atypical Hemolytic uremic syndrome \\
\hline
\end{tabular}

SLE-associated TTP-like MAHA. There are many cases of lupus complicated with TTP-like microangiopathic hemolytic anemia (MAHA). ${ }^{12,13}$ This condition in SLE is not preceded by diarrhea or low ADAMTS13 activity. Patients with TTP in SLE have an overall worse prognosis and usually present with advanced renal failure. ${ }^{14}$ Plasma exchange with fresh frozen plasma should be initiated immediately to offer survival benefit.

\section{Conclusion}

In a resource-constrained setting like ours, starting plasma exchange with fresh frozen plasma without waiting for the results of ADAMTS 13 activity level can offer a mortality benefit to patient of SLE presenting with TTP-like TMA.

\section{References}

1. Blum D, Blake G. Lupus-associated thrombotic thrombocytopenic purpura-like microangiopathy. World J Nephrol 2015 Nov 6;4(5): 528-531. DOI: 10.5527/wjn.v4.i5.528.

2. Chaudhury D, Qureshi A, Prasad S, et al. Catatonia - An unusual presenting clinical manifestation of systemic lupus erythematosus. Reumatol Clin 2017 JulAug;13(4):224-226. DOI: 10.1016/ j.reuma.2016.03.013.

3. Ainiala H, Loukkola J, Peltola J, et al. The prevalence of neuropsychiatric syndromes in systemic lupus erythematosus. Neurology 2001;57: 496-500. DOI: 10.1212/WNL.57.3.496.

4. Mak A, Ho RC, Lau CS, et al. Clinical implications of neuropsychiatric SLE. Adv Psychiatr Treat 2009;15:451-458. DOI: 10.1192/apt. bp.108.005785.

5. Grover S, Parakh P, Sharma A, et al. Catatonia in systemic lupus erythematosus: a case report and review of literature. Lupus 2013 May;22(6):634-638. DOI: 10.1177/0961203313486951.

6. Grover S, Singh A, Sarkar S, et al. SLE presenting with catatonia in an adolescent girl. J Pediatr Neurosci 2013 Sep;8(3):262-263. DOI: 10.4103/1817-1745.123715.

7. Pustilnik S, Trutia A. Catatonia as the presenting symptom in systemic lupus erythematosus. J Psychiatr Pract 2011;17:217-221. DOI: 10.1097/01.pra.0000398417.97609.2e.

8. Fink M, Taylor MA. Catatonia: A Clinician's Guide to Diagnosis and Treatment. New York, NY: Cambridge University Press; 2003.

9. Wang HY, Huang TL. Benzodiazepines in catatonia associated with systemic lupus erythematosus. Psychiatry Clin Neurosci 2006;60: 768-770. DOI: 10.1111/j.1440-1819.2006.01595.x.

10. Warrington TP, Bostwick JM. Psychiatric Adverse Effects of Corticosteroids. Mayo Clin Proc 2006;81:1361-1367. DOI: 10.4065/81.10.1361.

11. Fayyaz A, Igoe A, Kurien BT, et al. Haematological manifestations of lupus. Lupus Sci Med 2015 Mar 3;2(1):e000078. DOI: 10.1136/lupus2014-000078.

12. Abu-Hishmeh M, Sattar A, Zarlasht F, et al. Systemic Lupus Erythematosus Presenting as Refractory Thrombotic Thrombocytopenic Purpura: A Diagnostic and Management Challenge. A Case Report and Concise Review of the Literature. Am J Case Rep 2016 Oct 25;17:782-787. DOI: 10.12659/AJCR.898955.

13. Stricker RB. Two enigmas in one patient: TTP and SLE. South Med J 1995 Jul;88(7):795. DOI: 10.1097/00007611-199507000-00027.

14. Pattanashetti N, Anakutti H, Ramachandran R, et al. Effect of Thrombotic Microangiopathy on Clinical Outcomes in Indian Patients With Lupus Nephritis. Kidney Int Rep 2017 Apr 27;2(5):844-849. DOI: 10.1016/j.ekir.2017.04.008. 